\section{Onkologische Sicherheit der Laparoskopie beim Endometriumkarzinom}

\section{Originalpublikation}

Walker JL et al (2009) Laparoscopy compared with laparotomy for comprehensive surgical staging of uterine cancer: Gynecologic Oncology Group Study LAP2. J Clin Oncol 27:5331-5336

\section{Studiendesign}

Prospektive Multizenterstudie, in der Patientinnen mit einem klinisch Stadium-I- bis StadiumIIa-Tumor im Verhältnis 2:1 in einen Laparoskopiearm ( $n=1696$ Patientinnen) bzw. Laparotomiearm ( $\mathrm{n}=920$ Patientinnen) randomisiert wurden. Alle Patientinnen erhielten eine Hysterektomie, Adnexektomie, Entnahme einer Douglas-Zytologie sowie eine pelvine und paraaortale Lymphonodektomie. Primäres Studienziel war es, die Nichtunterlegenheit der LSK gegenüber der Laparotomie bezüglich Rezidivraten in der operativen Therapie uteriner Karzinome zu zeigen. Definiert wurde dieses Ziel, indem es eine um nicht mehr als 40\% höhere Rezidivrate nach LSK vs. Laparotomie zu zeigen galt.

\section{Ergebnisse}

Nach einem durchschnittlichen Follow-up von 59 Monaten lebten 2181 Patientinnen, 309 hatten ein Rezidiv (210 im LSK-Arm und $99 \mathrm{im}$ Laparotomiearm) und 350 Patientinnen waren verstorben, wobei dies 229 nach LSK und 121 nach Laparotomie waren. Die geschätzte Hazard Ratio

Urologe 2013 $\cdot 52: 1004$

DOI 10.1007/s00120-013-3235-0

(c) Springer-Verlag

Berlin Heidelberg 2013

Redaktion

H. Rübben, Essen

für die LSK vs. Laparotomie lag gemäß protokollspezifischer Definition der Nichtunterlegenheit bei 1,14 (untere 90\%-Grenze: 0,92 sowie obere 95\%-Grenze: 1,46). Die aktuellen Rezidivraten waren indes wesentlich niedriger als erwartet. Die Dreijahresrezidivrate betrug nach Laparoskopie 11,4\% und nach Laparotomie 10,2\%, was einer Differenz von 1,14\% entspricht (untere 90\%-Grenze: $-1,28$ sowie obere $95 \%$-Grenze: $4,0)$. Das geschätzte Fünfjahresgesamtüberleben war mit $89,8 \%$ in beiden Therapiearmen identisch.

\section{Kommentar \\ Die Studie klärt noch verbliebene privation in Prostate Cancer. N Engl J Med 368:1326-1334} offene Fragen in der endoskopischen Therapie des Endometriumkarzinoms. Während die Kurz- und Langzeitmorbidität nach einer LSK durch mehrere prospektiv-randomisierte Studien belegt und bestätigt worden waren, ist die onkologische Sicherheit des Verfahrens stets ein offener Punkt vieler Diskussionen geblieben.

Walker et al. konnten zeigen, dass Patientinnen nach einer LSK nahezu identische Gesamtüberlebensraten bzw. keine wesentlich höheren Rezidivraten als nach einer Laparotomie haben. Diese Ergebnisse sind zwar in den bisherigen Studien meist für Frühkarzinome gezeigt worden, allerdings wird ein Großteil der Endometriumkarzinome im Frühstadium erkannt. Somit betreffen diese Ergebnisse einen Großteil unseres Patientenguts.

Ein weiterer Diskussionspunkt war und ist die onkologische Sicherheit der LSK nicht nur bei Low-Risk-Karzinomen (also endometroiden G1- oder G2-Karzinomen), sondern bei G3- bzw. nichtendometroiden Karzinomen. Walker et al. haben in

\section{Originalpublikation}

Hussain M et al (2013) Intermittent versus Continuous Androgen De-

Intermittierende Androgendeprivation (ADT) kann helfen, unerwünschten Folgen kontinuierlichen Hormonentzugs vorzubauen. Erkauft wird dies aber möglicherweise mit einer verkürzten Überlebenszeit.

Für eine Studie wurden die Daten von 1.535 Patienten mit metastasiertem, hormonsensiblem Prostatakarzinom ausgewertet. Alle Männer hatten eine siebenmonatige Dauertherapie erhalten. Probanden mit PSA-Wert von höchstens $4 \mathrm{ng} / \mathrm{ml}$ wurden danach randomisiert auf zwei Gruppen verteilt. 765 wurden kontinuierlich, 770 intermittierend mit einem GnRH-Analogon (Goserelin) und einem Antiandrogen (Bicalutamid) behandelt. Sobald der PSA-Spiegel auf den Ausgangswert zu Beginn der Studie oder auf $20 \mathrm{ng} / \mathrm{ml}$ gestiegen war, setzte die Androgendeprivation in der zweiten Gruppe wieder ein. War die Konzentration nach weiteren sieben Monaten wieder auf $4 \mathrm{ng} / \mathrm{ml}$ oder darunter gesunken, wurde der Entzug erneut ausgesetzt. ihrem Kollektiv eben diese Subgruppen mituntersucht. Diese Subgruppenanalyse war zwar kein vorab definierter Studienendpunkt, auch waren die Subgruppen nicht ausreichend gepowert, aber die Autoren konnten keinen signifikanten Rezidivunterschied in Abhängigkeit vom Tumorgrading oder histologischen Typ nachweisen.

Ein weiterer wichtiger Aspekt, den die Studie berücksichtigt, ist die Metastasierungsrate im Be-

reich der LSK-Einstichstellen. Diese GOG-Studie zeigt, dass die Inzidenz für eine solche $\mathrm{Me}$ tastasierung bei lediglich $0,24 \%$ liegt ( $\mathrm{n}=4$ Patientinnen von 1696 laparoskopischen Operationen), wobei bei 3 der 4 Patientinnen letztendlich fortgeschrittene Stadium-III- und Stadium-IVKarzinome vorlagen.

Quelle: Onkologe 18:714-717

\title{
Dr. Ingolf Juhasz-Böss
}

\section{Verkürzt intermittierende ADT das Leben?}

Männer unter intermittierender Deprivation berichteten nach drei Monaten über eine bessere Lebensqualität, gemessen an psychischer Gesundheit, Erektionsfunktion und Libido. Die Unterschiede waren aber nur gering.

Ein Hauptziel der Studie war es, die Nicht-Unterlegenheit der intermittierenden gegenüber der kontinuierlichen Androgendeprivation hinsichtlich des Überlebens der Patienten zu zeigen. Diess misslang. Die mediane Überlebenszeit in Gruppe eins betrug 5,8 Jahre, in Gruppe zwei 5,1 Jahre. Das Sterberisiko während der Nachbeobachtungszeit (median 9,8 Jahre) war unter intermittierender Deprivation um 10\% erhöht. Diese Steigerung unterschritt zwar die definierte Schwelle von $20 \%$ für die NichtInferiorität. Das $90 \%$-Konfidenzintervall jedoch reichte von $-1 \%$ bis $+23 \%$, das $95 \%$-Intervall von $-3 \%$ bis $+25 \%$. Damit ließ sich weder die Nicht-Inferiorität der intermittierenden Therapie noch ihre Unterlegenheit statistisch sicher belegen.

Quelle: Uro-News 17:48

\section{Dr. Robert Bublak}

